\title{
Movement of materials in gyroscopic-type mixer
}

\author{
Konstantin Anatolyevich Yudin \\ Department of mechanical equipment \\ Belgorod State Technological University named after \\ V.G. Shoukhov \\ BSTU named after V.G. Shoukhov \\ Belgorod, Russia \\ 308012, Kostukov St., 46 \\ kyudin@mail.ru
}

\author{
Vasily Stepanovich Bogdanov \\ Department of mechanical equipment \\ Belgorod State Technological University named after \\ V.G. Shoukhov \\ BSTU named after V.G. Shoukhov \\ Belgorod, Russia, \\ 308012, Kostukov St., 46
}

\author{
Andrey Nikolayevich Degtyar \\ Department of structural resistance and engineering \\ mechanics \\ Belgorod State Technological University named after \\ V.G. Shoukhov \\ BSTU named after V.G. Shoukhov \\ Belgorod, Russia, \\ 308012, Kostukov St., 46
}

\author{
Artyom Sergeevich Stovpenko \\ Department of mechanical equipment \\ Belgorod State Technological University named after \\ V.G. Shoukhov \\ BSTU named after V.G. Shoukhov \\ Belgorod, Russia, \\ 308012, Kostukov St., 46
}

\begin{abstract}
Peculiarities of development of gyroscopic-type mixers were considered. The expediency of development of such mixers is presented. The literature review on this subject was performed. Author's kinematic scheme and a variant of the laboratory plant of the mixer (three-dimensional model), describing the influence on the material, mixed in the mixing chamber realtively two inteperpendicular horizontal axes, were presented. The mixing chamber is rotated by means of conic, chain and cylindrical gearings. The emerging complex spacial motion of material particles can be regulated by the frequency transformer and selection of corresponding cogwheels. The part of the methodology on defining the path of material particles in the mixing chamber is presented. The spatial task is solved mathematically. Preliminary experimental studies were conducted. A central composite orthogonal plan of the fractional factor experiment was chosen. Four parameters were chosen as input factors. An example of machine realisation of the task by means of SolidWorks Flow Simulation was presented. Conclusions about the work were made.
\end{abstract}

Keywords - flow paths inside mixing chamber, batch mixing unit, chamber rotation about two mutually horizontal axes, train gears.

\section{INTRODUCTION}

Significant energy expenditures and sizes of the used equipment in industry of construction materials and in allied industries compel the application of innovation approaches. The problem direction is processing materials. Specificity of equipment leads to the necessity of enhancing the effectiveness of its exploitation, taking into account the methods of material processing. The mixing equipment is of primary importance among the directions of material processing [1-3]. One of the innovation approaches is the use of two-directional influence on the mixed material in batch mixing units, which implies enhancement of operating effectiveness of the latter $[4,5]$.

\section{Main Part}

It is possible to supplement the existing classification of mixers with batch mixing units, using the elements of gyroscopic effect. This implies two-directional influence on the mixing chamber with material, including chamber rotation relatively two mutually perpendicular (vertical and horizontal, two horizontal) axes [6-8].

The example of using such approach is modernisation of batch mixing units for dry construction mixtures. Besides, the importance of mixers during production of gas-silicate products is significant.

As is well known, the most important factor, influencing the cell structure of the cellular-concrete mixture and readymade concrete, is uniformity of distribution of a small amount $(0,6 \ldots 0,7 \mathrm{~kg} / \mathrm{m} 3)$ of gasifier (aluminium powder) in the whole volume of the mixture.

The used gas-concrete mixers are equipped with a vertical blade shaft, rotated by the driver. Besides, to intensify the mixing, the mixture is subjected to vibration by means of suspended vibrators [9].

The proposed apparatus for mixing quarzt sand, cement, lime and aluminium powder influences the mixed material in two mutually perpendicular horizontal directions [10]. Thus, the mixing chamber of the gas-concrete mixer is subjected to rotation around two mutually perpendicular horizontal axes, 
which implies using the elements of gyroscopic effect in batch mixing units.

The technical result of creation of the apparatus is enhancing the effectiveness of mixing materials.

Let us present the kinematic scheme of the proposed apparatus (Figure 1).

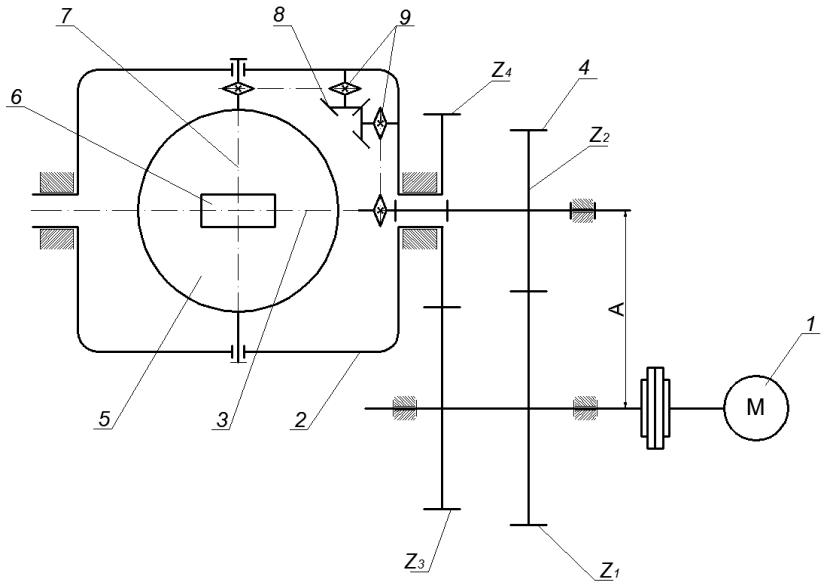

Fig. 1. Kinematic scheme of apparatus for mixing materials with rotation of chamber around two mutually perpendicular horizontal axes:

1 - driver; 2 - rotating carrier; 3 - horizontal axis; 4 - cylindrical gearing; 5 mixing chamber; 6 - charging hatch; 7 - second horizontal axis; 8 - conical gearing; 9 - chain gearing

The technical result is achieved by the device for mixing materials, containing a mixing chamber of spherical shape, mounted on the carrier, rotated around the horizontal axis and the rotary drive. The conical gearing used for rotating the mixing chamber around the second horizontal axis is supplemented by the chain gearing [5].

The presence of the chain gearing allows reducing sizes of conical gears of transmission, rotating the mixing chamber, which will reduce material consumption of the apparatus construction for mixing materials.

Let us demonstrate a variant of realisation of the batchtype mixer taking into account two-directional influence on the mixed material inside the mixing chamber relatively two mutually perpendicular horizontal axes.

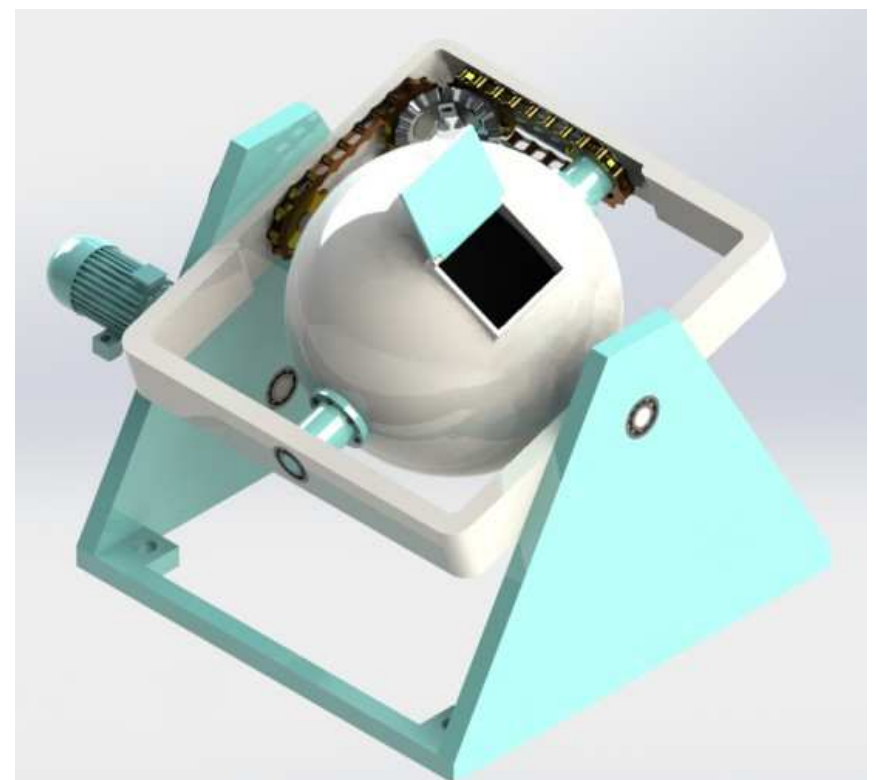

Fig. 2. Three-dimensional model of apparatus for mixing materials

On the one hand, centrifugal forces are conditioned by rotation of the carrier with the spherical mixing chamber, fixed on it, relatively the horizontal axis. On the other hand, centrifugal forces are conditioned by the rotation of the mixing chamber around the second horizontal axis. Superposition of forces makes particles move along the complex path.

The operating mode of the apparatus for mixing materials is periodical. The mixing chamber of spherical shape must have a small diameter to avoid extreme overloads on bearing assemblies.

A spherical shape of the mixing chamber and the variation of rotational frequencies are expedient for elimination of dead loading zones and increasing the intensity of mixing since, with a certain ratio of frequencies, a motion path of loading, optimal for this material, appears. All this facilitates the increase of the area of contacting surfaces, the number of interactions and material diffusion. The phase of work is terminated by the stoppage of the rotational drive through the certain interval of time and unloading the mixing chamber through the hatch.

Prepared mixtures can be effectively unloaded by installing the unloading assembly, combined with the portal, under the mixing chamber.

Let us demonstrate the part of the methodology on determination of the motion path of material particles, solving the spacial task. Specifics of influence on the mixed material leads to complication of the mathematical apparatus for such task.

Let us consider that the material particle moves inside the mixing chamber only under the influence of gravity [6]:

$$
m_{i} \ddot{\bar{r}}_{i}=\bar{G}_{i}
$$

where $m_{i}$ - mass of the $i$-th particle, $\bar{G}_{i}-$ weight of the $i$-th particle. 
Taking into account that $G_{i}=m_{i} g$ and integrating equation (1) twice, one will obtain:

$$
\left\{\begin{array}{c}
\ddot{\bar{r}}_{i}=-g \bar{k} \\
\ddot{\bar{r}}_{i}=-g \bar{k} t+\bar{V}_{i 0} \\
\ddot{\bar{r}}_{i}=-g \bar{k} \frac{t^{2}}{2}+\bar{V}_{i 0} t+\bar{r}_{i 0} .
\end{array}\right.
$$

In the general case, in the absence of contact between the chosen ball-shaped particle and other particles and the lining of the mixing chamber, the motion of mass centres of each particle is described by the equation:

$$
\bar{r}_{i}=\bar{r}_{i 0}+\bar{V}_{i o t}+\frac{\bar{g} t^{2}}{2}
$$

Equation (3) in projections will acquire the following form:

$$
\left\{\begin{array}{c}
x_{i}=x_{i 0}+V_{i x o} t \\
y_{i}=y_{i 0}+V_{i y o} t \\
z_{i}=z_{i 0}+V_{i x o} t-\frac{g t^{2}}{2} .
\end{array}\right.
$$

For each i-th particle, it is possible to determine time $t_{i b}$ of its motion along the parabolic trajectory up to collision with the mixing chamber. A limiting condition is of the form:

$$
x_{i}^{2}+y_{i}^{2}+z_{i}^{2}=\left(R_{b}-r_{s}\right)^{2}
$$

where $R_{b}$ - radius of the mixing chamber, $r_{s}-$ particle radius.

Equation (5), taking into account (4), acquires the form:

$$
\begin{aligned}
& \left(x_{i 0}+V_{i x o} t_{i b}\right)^{2}+\left(y_{i 0}+V_{i y o} t_{i b}\right)^{2} \\
& +\left(z_{i 0}+V_{i z o} t_{i b}-\frac{g}{2} t_{i b}^{2}\right)^{2}= \\
& \left(x_{i 0}+V_{i x o} t_{i b}\right)^{2}+\left(y_{i 0}+V_{i y o} t_{i b}\right)^{2} \\
& +\left(z_{i 0}+V_{i z o} t_{i b}-\frac{g}{2} t_{i b}^{2}\right)^{2}= \\
& \quad=\left(R_{b}-r_{s}\right)^{2}
\end{aligned}
$$

Equation (6) is the equation of the 4-th order.

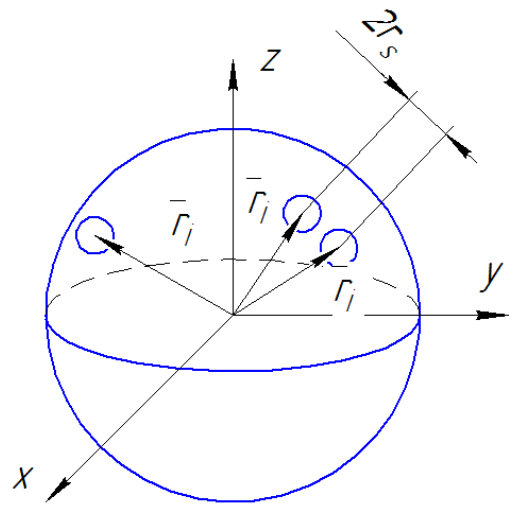

Fig. 3. Material particles inside the mixing chamber
Equation 6 has four real roots. Since initially the material particle is inside the mixing chamber, one root will be always negative, and the necessary root will be minimally positive. The variants of particle movement can be interpreted graphically (Figure 4).

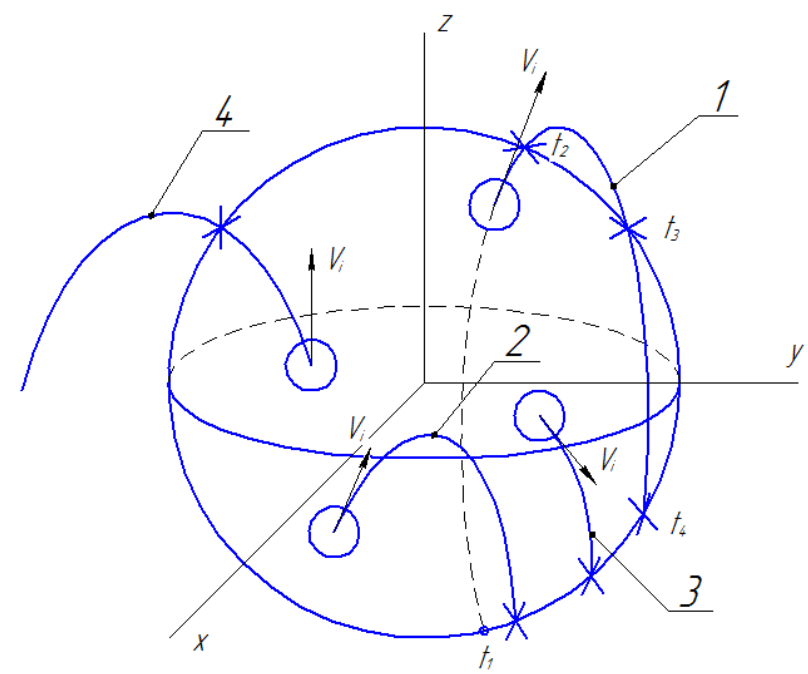

Fig. 4. Variants of particles' motion

Similarly, it is possible to calculate the time before particle collision $\left|\bar{r}_{i}-\bar{r}_{j}\right|=2 r_{s}$, which in the scalar form looks as follows:

$$
\begin{gathered}
\left(x_{i 0}+V_{i x o} t_{i j s}-x_{j 0}-V_{j x o} t_{i j s}\right)^{2} \\
+\left(y_{i 0}+V_{i y o} t_{i j s}-y_{j 0}\right. \\
\left.-V_{j y o} t_{i j s}\right)^{2}+ \\
+\left(z_{i 0}+V_{i z o} t_{i j s}-\frac{g}{2} t_{i j s}^{2}-z_{j 0}-V_{j z o} t_{i j s}+\frac{g}{2} t_{i j s}^{2}\right)^{2} \\
=\left(R_{b}-r_{s}\right)^{2}
\end{gathered}
$$

Let us consider the collision of material particles with the mixing chamber. Let us suppose that the particle position during collision does not change and the center-of-mass velocity of the particle changes instantly.

Let us write for the particle the theorems of the change of the motion amount and of the change of the angular momentum amount:

$$
\left\{\begin{array}{l}
m \bar{V}_{1}-m \bar{V}_{0}=\bar{S}_{n}+\bar{S}_{\tau} ; \\
J\left(\bar{\varpi}_{1 \mathrm{II}}-\bar{\varpi}_{0 \mathrm{I}}\right)=m_{z}\left(\bar{S}_{n}\right)+m_{z}\left(\bar{S}_{\tau}\right) ; \\
J\left(\bar{\varpi}_{1 \mathrm{II}}-\bar{\varpi}_{0 \mathrm{II}}\right)=m_{y}\left(\bar{S}_{n}\right)+m_{y}\left(\bar{S}_{\tau}\right) .
\end{array}\right.
$$

where $J=\frac{2 \cdot m r^{2}}{5}-$ moment of particle inertia relatively the axis, passing through its mass centre;

$\omega_{1}, \omega_{0}-$ angular velocities after contact and before contact;

$\mathrm{S}_{\mathrm{n}}$ - impulse of the force of normal pressure;

$\mathrm{S}_{\tau}$ - impulse of the force of normal pressure;

$\mathrm{V}_{1}, \mathrm{~V}_{0}$ - particle velocity after contact and before contact. 
Let us project equation (8) on the coordinate axis, connected to point $\mathrm{B}$ on the inner surface of the mixing chamber.

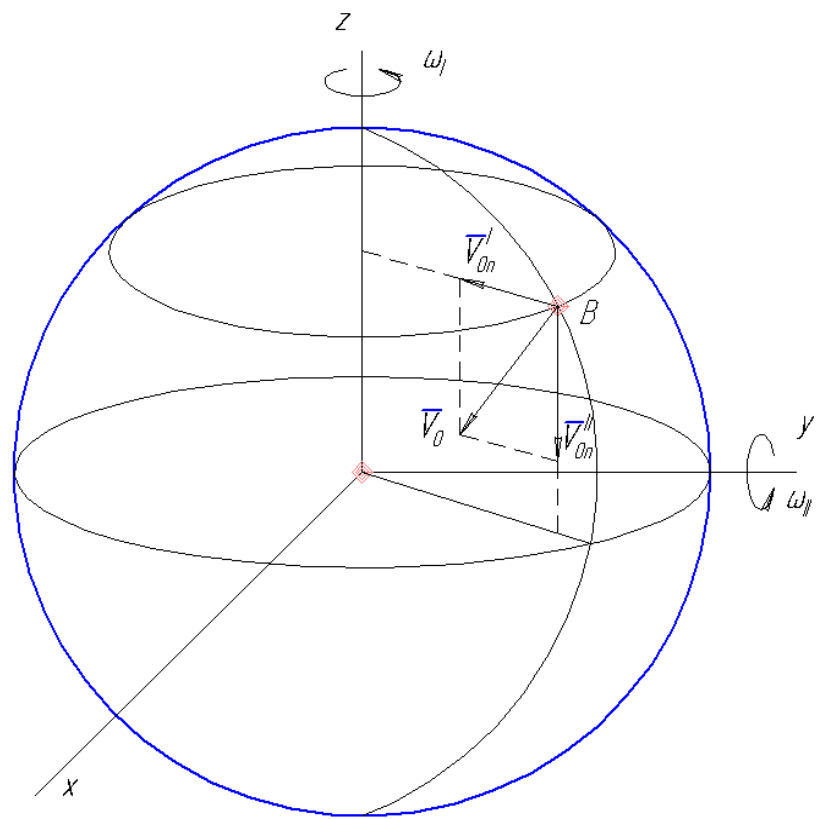

Fig. 5. Velocity in point B before collision

$$
\left\{\begin{array}{l}
m \bar{V}_{1 n}-m \bar{V}_{0 n}=\left(\bar{S}_{1 n}^{I}\right) ; \\
m \bar{V}_{1 \tau_{1}}-m \bar{V}_{0 \tau_{1}}=S_{1 \tau_{1}} ; \\
m \bar{V}_{1 \tau_{2}-m \bar{V}_{0 \tau_{2}}=S_{1 \tau_{2}} ;} ; \\
J \varpi_{I}=\sum m_{z}\left(\bar{S}_{i}\right) ; \\
J \varpi_{I I}=\sum m_{y}\left(\bar{S}_{i}\right),
\end{array}\right.
$$

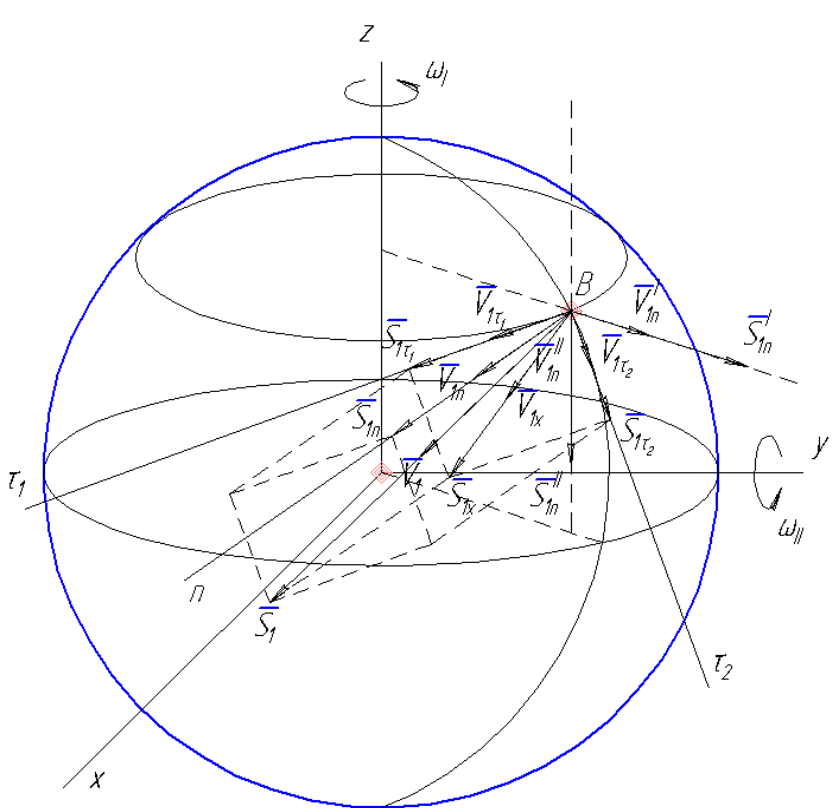

Fig. 6. Velocity in point B after collision
As a result of solving system (9), let us determine the components of velocity V1. Achievement of the technical result was confirmed by experimental studies [11]. Following the theory of planning experiments, let us dwell on the central composite orthogonal plan of the fractional factorial experiment. As input parameters, the following was chosen: [phi] - load factor of the mixing chamber; $\mathrm{n}$ - rotation frequency, $\mathrm{s}^{-1}$; [rho] - coarseness of particles of the feed material, $\mathrm{m} ; \mathrm{t}-$ time of mixing the feeding mixture, $\mathrm{s}$.

The obtained experimental data should be supplemented by the machine experiment with specific software, for example SolidWorksFlowSimulation. The modern application "SolidWorks" is meant for hydro-gas-dynamic analysis.

For example, with the rotational frequency equal to $0.3 \mathrm{~s}^{-1}$, it is possible to obtain the following diagrams 7-9.

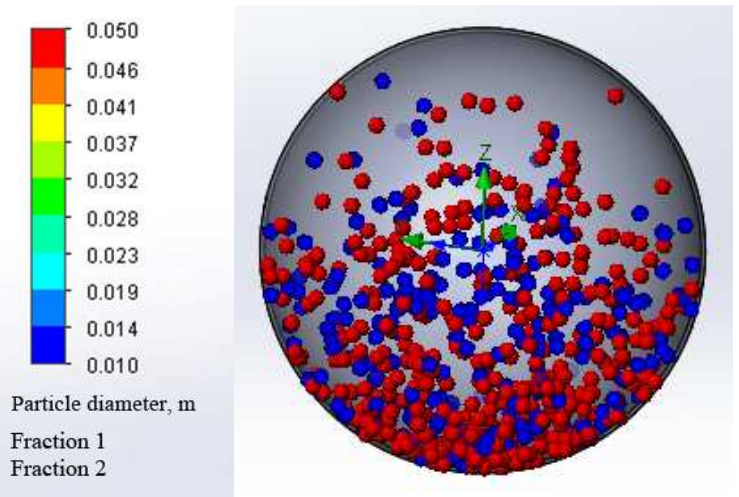

Fig. 7. Collision density of material particles

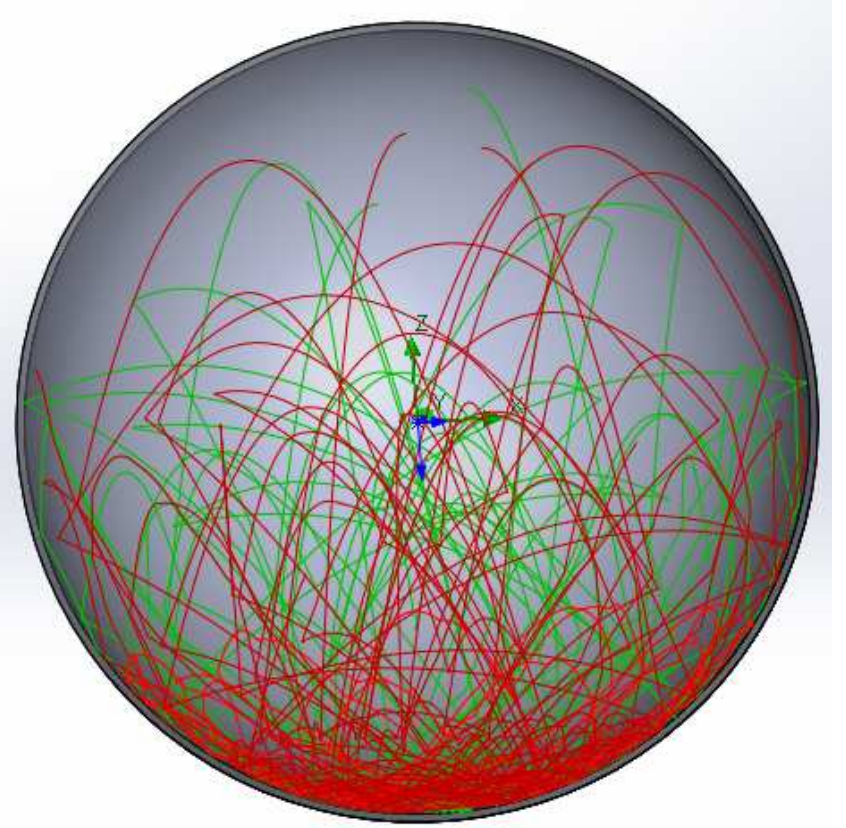

Fig. 8. Particle motion path 

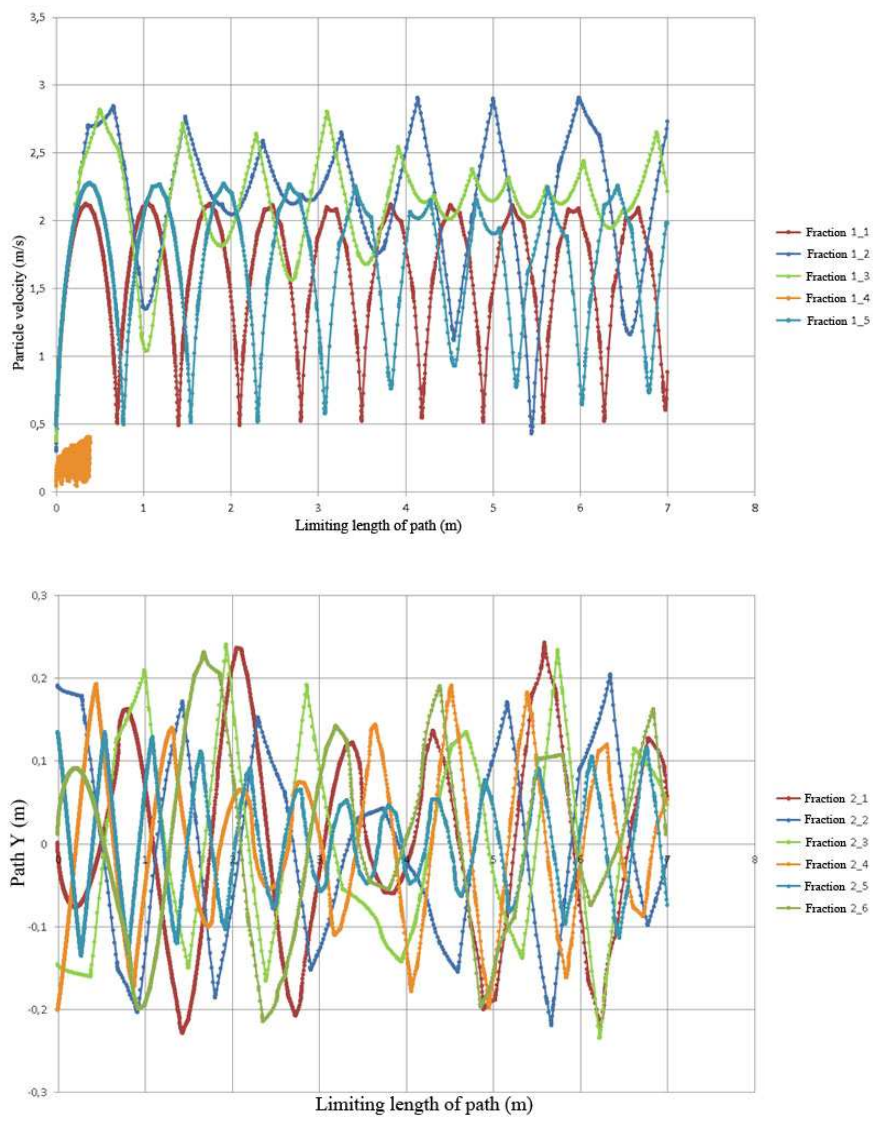

Fig. 9. Diagrams of particle motion analysis

Findings about the possibility of using such mixtures can be made as a result of comparing the parameters, calculated by means of the developed methodology, of mixing processes and production data.

The task of optimizing constructional-process variables of mixers is realised, for example, by the successive simplexmethod with the use of the mathematical model of the multiphase cycle of mixture motion.

\section{CONCLUSION}

1. Analysis of the operation of the existing mixing equipment and theories of the mixing process showed the necessity of developing the methodology of determining the optimal parameters of mixtures' interaction in the apparatuses, realising two-directional influence on the material.

2. Experimental plants were created and the necessary number of experiments was conducted by the central composite orthogonal plan.

3. Based on the mathematical model of the apparatus for mixing materials, optimal paths of particle motions of the mixture for its effective homogenization were revealed.

4. For the construction of the apparatus for mixing materials, developed taking into account the proposed methodology, author' certificates of the useful model were received.

\section{Acknowledgment}

The work is realized in the framework of the Program of flagship university development on the base of Belgorod State Technological University named after V.G. Shoukhov, using equipment of the High Technology Center at BSTU named after V.G. Shoukhov.

\section{References}

[1] V.S. Bogdanov, R.R. Sharapov, Yu.M. Fadin, I.A. Semikopenko, N.P. Nesmeyanov, V.B. Gerasimenko, Basics of calculation of machines and equipment of enterprises of construction materials and products, Manual, Stariy Oskol: TNT, 2013.

[2] V.S. Bogdanov, Processes in production of construction materials and products. Manual, Belgorod: Vezelitsa, 2007.

[3] S. P. Deolalkar, Handbook for designing cement plants, CRCPress.

[4] United States Patent 4326428, Two degrees of freedom rate gyroscope, date views 27.04.1982, Retriewed from: http://www.freepatentsonline.com/4326428.html

[5] K.A. Yudin, A.N. Degtyar, "Peculiarities of material motion in gyroscopic-type mixer," Bulletin of BSTU named after V.G. Shukhov, 2015, pp. 108-111.

[6] John J. Uicker, R. Pennock Gordon, E. Joseph Shigley, Theory of Machines and Mechanisms, Oxford University Press, 2009.

[7] K. A. Yudin, L.A. Kuschev, I. N. Savkin and A. N. Negrun, "Modeling mixers of gyroscopic type," Middle-East Journal of Scientific Research, 17 (8), pp. 1125-1129.

[8] I. Karimov, Theoretical mechanics, Retriewed from: http://www.teoretmeh.ru/.

[9] Yu.P. Gorlov, Technology of heat-insulating and acoustic materials and products, Vysshaya shkola, 1989.

[10] V.S. Bogdanov, K.A. Yudin, I.N. Savkin, A.N. Negrun, Apparatus for mixing materials", applicant and patent holder, Patent №144695 RF MPK7B22C5, Bull. №24, pp. 4.

[11] P.V. Trusov, Introduction into mathematical modelling, Manual. Moscow: Logos, 2005. 